\title{
THE PROBLEM OF INTEGRATION IN FINITE TERMS
}

\author{
BY \\ ROBERT H. RISCH( $\left.{ }^{1}\right)$
}

This paper deals with the problem of telling whether a given elementary function, in the sense of analysis, has an elementary indefinite integral.

In $\S 1$ of this work, we give a precise definition of the elementary functions and develop the theory of integration of functions of a single varia: $\therefore$. By using functions of a complex, rather than a real variable, we can limit ourselves to exponentiation, taking logs, and algebraic operations in defining the elementary functions, since $\sin , \tan ^{-1}$, etc., can be expressed in terms of these three. Following Ostrowski [9], we use the concept of a differential field. We strengthen the classical Liouville theorem and derive a number of consequences.

$\$ 2$ uses the terminology of mathematical logic to discuss formulations of the problem of integration in finite terms.

$\$ 3$ (the major part of this paper) uses the previously developed theory to give an algorithm for determining the elementary integrability of those elementary functions which can be built up (roughly speaking) using only the rational operations, exponentiation and taking logarithms; however, if these exponentiations and logarithms can be replaced by adjoining constants and performing algebraic operations, the algorithm, as it is presented here, cannot be applied.

The man who established integration in finite terms as a mathematical discipline was Joseph Liouville (1809-1882), whose work on this subject appeared in the years 1833-1841. The Russian mathematician D. D. Mordoukhay-Boltovskoy (1876-1952) wrote much on this and related matters. The present writer received his introduction to this subject through the book [10] by the American J. F. Ritt (1893-1951).

The reader need only be familiar with some standard facts from algebra and complex analysis in order to understand this paper. Some basic results from differential algebra are used, but they are explicitly stated and references are given for their proofs.

1. Liouville theory of elementary functions. A field $\mathscr{D}$ with a unary operation $d / d z$ is said to be a differential field iff for any $a, b$ in $\mathscr{D}$ :

$$
\begin{aligned}
\frac{d}{d z}(a+b) & =\frac{d}{d z} a+\frac{d}{d z} b \\
\frac{d}{d z}(a \cdot b) & =a \cdot \frac{d}{d z} b+b \cdot \frac{d}{d z} a .
\end{aligned}
$$

Received by the editors March 28, 1967 and, in revised form, April 22, 1968.

(1) Present address: T. J. Watson, Research Center, Yorktown Heights, New York. 
We will also write $(d / d z) a$ as $a^{\prime}$. We will always assume that the characteristic of our field is 0 . A differential field containing $\mathscr{D}$ is a differential extension of $\mathscr{D}$. An isomorphism of the differential fields $\mathscr{D}_{1}$ and $\mathscr{D}_{2}$ which preserves the differentiation operation is called a differential isomorphism. $K=\{a \in \mathscr{D}:(d / d z) a=0\}$ is a differential field and is called the constant field of $\mathscr{D}$.

Let $U$ be a differential field extension of $\mathscr{D}$ having the property that for any finitely generated differential extension $\mathscr{F}$ of $\mathscr{D}$, there is a differential isomorphism of $\mathscr{F}$ into $U$, leaving $\mathscr{D}$ fixed; then $U$ is a universal extension of $\mathscr{D}$.

It is proved in [4, pp. 768-771], that every differential field has a universal extension. There a stronger definition of universal extension is used. We will denote the constant field of $U$ by $C$.

It may be of help to think of $\mathscr{D}$ as a field of meromorphic functions, that is finitely generated over the rational numbers, on some region $A$ of the complex plane or a Riemann surface, of $U$ as consisting of all functions which are meromorphic on some subregion of $A$, and of $C$ as the complex numbers.

For $\theta \in U, \theta$ and $\mathscr{D}(\theta)$ are said to be simple elementary over $\mathscr{D}$ iff one of the following conditions holds:

(1) $\theta$ is algebraic over $\mathscr{D}$.

(2) There is an $f$ in $\mathscr{D}, f \neq 0$, such that $f^{\prime}=f \theta^{\prime}$.

This situation will be abbreviated as $\theta=\log f$.

(3) There is an $f$ in $\mathscr{D}$ such that $\theta^{\prime}=\theta f^{\prime}$. Here we write $\theta=\exp f$.

Note that $\mathscr{D}(\theta)$ is, in an obvious way, a differential field. Also observe that any $c \in C$ is simple elementary over $\mathscr{D}$. If we have for $\theta, \psi \in U, \theta=\log f$ and $\psi=\log f$ or $\theta=\exp f$ and $\psi=\exp f$, then $\psi=\theta+c$, respectively $\psi=c \theta$ for some $c \in C$.

In cases (2) and (3), if the constant field of $\mathscr{D}(\theta)=K$ and $\theta$ is transcendental over $\mathscr{D}$, then $\theta$ is said to be a monomial over $\mathscr{D}$. It is easy to show that if $\theta=\log f$ or $\theta=\exp f$ for $f \in \mathscr{D}$ then $\theta$ is not a monomial over $\mathscr{D}$ iff there is a $c \in \mathscr{D}(\theta) \cap C$ such that $\theta$ is algebraic over $\mathscr{D}(c)$. A useful example here is $\mathscr{D}=Q\left(z, e^{z}\right)$, where $Q=$ rationals and $z$ is the identity function on a region $A$. If $\theta$ and $\psi$ are two different logarithms of $e^{z}$ (say $z$ and $z+2 \pi i$ ), then $\psi$ is not a monomial over $\mathscr{D}(\theta)$.

Let $\mathscr{F}=\mathscr{D}\left(\theta_{1}, \ldots, \theta_{n}\right)$, each $\theta_{i}$ being simple elementary over $\mathscr{D}\left(\theta_{1}, \ldots, \theta_{i-1}\right)$, $i=1, \ldots, n$. Then $\mathscr{F}$ and any $g \in \mathscr{F}$ is said to be elementary over $\mathscr{D} . \mathscr{F}$ and any $g \in \mathscr{F}$ are regular elementary over $\mathscr{D}$ iff each $\theta_{i}$ is a monomial or algebraic over $\mathscr{D}\left(\theta_{1}, \ldots, \theta_{i-1}\right)$.

We now turn to a proof and sharpening of the basic Liouville theorem on integration in finite terms. This result is usually proved by an analytic technique which was introduced by Liouville in [7]. In Mordoukhay-Boltovskoy's book [8], the technique of partial fractions in monomial extensions was first used to study elementary integration. That the partial fraction method could be used to prove Liouville's theorem itself was pointed out by Rosenlicht in [11].

First we study the differentiation of certain types of elements of monomial extension fields. Let $\mathscr{D}$ be a differential field, $\theta$ a monomial over $\mathscr{D}$. 
(a) Let $P \in \mathscr{D}[\theta], \quad P=A_{n} \theta^{n}+A_{n-1} \theta^{n-1}+\cdots+A_{0}, \quad A_{i} \in \mathscr{D}, \quad A_{n} \neq 0$.

Case 1. $\theta=\log f$. Here $P^{\prime}=A_{n}^{\prime} \theta^{n}+\left(A_{n-1}^{\prime}+n A_{n}\left(f^{\prime} \mid f\right)\right) \theta^{n-1}+\cdots+\left(A_{0}^{\prime}+A_{1}\left(f^{\prime} \mid f\right)\right)$. If $A_{n}^{\prime}=0$, then $A_{n-1}^{\prime}+n A_{n}\left(f^{\prime} \mid f\right) \neq 0$; for if it were, then $A_{n-1}+n A_{n} \theta=$ constant and $\theta$ would not be a monomial over $\mathscr{D}$. Here we use the remark following the definition of simple elementary above. Thus the derivative of a polynomial of degree $n$ in $\theta=\log f$ is a polynomial of degree $n-1$ if its leading coefficient is a constant. It is a polynomial of degree $n$ if the leading coefficient is nonconstant.

Case 2. $\theta=\exp f$. Then

$$
P^{\prime}=\left(A_{n}^{\prime}+n f^{\prime} A_{n}\right) \theta^{n}+\left(A_{n-1}^{\prime}+(n-1) f^{\prime} A_{n-1}\right) \theta^{n-1}+\cdots+\left(A_{1}^{\prime}+f^{\prime} A_{1}\right) \theta+A_{0}^{\prime} .
$$

$A_{n}^{\prime}+n f^{\prime} A_{n} \neq 0$, for if it were then $\theta^{-n}=$ constant $\times A_{n}$ and $\theta$ is not a monomial over $\mathscr{D}$. Thus, the derivative of $P$ is of the same degree as $P$.

(b) Let $P, Q$ be the elements of $\mathscr{D}[\theta], Q$ monic and irreducible in $\theta, p=$ degree $P$ $<q=$ degree $Q$

$$
\left(P / Q^{n}\right)^{\prime}=-n P Q^{\prime} / Q^{n+1}+P^{\prime} / Q^{n} \text {. }
$$

Case 1. $\theta=\log f$. By (a), degree $Q^{\prime}=q-1$. Thus, $Q \nmid-n P Q^{\prime}$, for if it did, it would have to divide $P$ or $Q^{\prime}$. Thus, the partial fraction decomposition of $\left(P / Q^{n}\right)^{\prime}$ is of the form $R / Q^{n+1}+S / Q^{n}, R \neq 0$.

Case 2. $\theta=\exp f$. Suppose $Q \mid-n P Q^{\prime}$. Since $Q \nmid P$ we have that $Q \mid Q^{\prime} . Q=\theta^{q}+\cdots$, so $Q^{\prime}=q f^{\prime} \theta^{q}+\cdots$, so $Q^{\prime}=q f^{\prime} Q$. Therefore, $Q=\left(e^{f}\right)^{q}=\theta^{q}$. Since $Q$ is irreducible, $q=1$ and $P \in \mathscr{D}$.

Thus, the partial fraction decomposition of $\left(P / Q^{n}\right)^{\prime}$, unless $Q=\theta$, is of the form $R / Q^{n+1}+S / Q^{n}, R \neq 0$. If $Q=\theta$, then it is of the form $\left(P^{\prime}-n f^{\prime} P\right) / Q^{n}, P^{\prime}-n f^{\prime} P \neq 0$.

(c) Let $P \in \mathscr{D}[\theta], P$ monic, degree $P=p$.

Case 1. $\theta=\log f$. Then $(\log P)^{\prime}=P^{\prime} / P$ where degree $P^{\prime}=p-1$.

Case 2. $\theta=\exp f$. Then $(\log P)^{\prime}=P^{\prime} \mid P=N / P+p f^{\prime}$ where $N=P^{\prime}-p f^{\prime} P . N=0$ in case $P=\theta$ and otherwise $N$ is a polynomial of degree less than $p$.

Theorem of Liouville-First Statement. Let $\mathscr{D}$ be a differential field, $\mathscr{F}$ elementary over $\mathscr{D}$. Suppose $\mathscr{D}$ and $\mathscr{F}$ have the same constant field $K$. Let $g \in \mathscr{F}$, $f \in \mathscr{D}$ with $g^{\prime}=f$. Then $g=v_{0}+\sum_{i=1}^{k} c_{i} \log v_{i}$ where $v_{0}, v_{i}$ are els of $\mathscr{D}$ and $c_{i}$ els of $K$.

Proof. $\mathscr{F}$ is regular elementary over $\mathscr{D} ; \mathscr{F}=\mathscr{D}\left(\theta_{1}, \ldots, \theta_{n}\right)$, each $\theta_{i}$ being a monomial or algebraic over $\mathscr{D}\left(\theta_{1}, \ldots, \theta_{i-1}\right)$. We use induction on $n$. Suppose $n=0$, then $\mathscr{F}=\mathscr{D}$ and $g=v_{0}$ where $v_{0} \in \mathscr{D}$. Assume we have proven the theorem for $n-1$. Then $g=w_{0}+\sum_{i=1}^{k} d_{i} \log w_{i}$, where $w_{0}, w_{i}$ els $\mathscr{D}\left(\theta_{1}\right)$, and $d_{i}$ els $K$.

Case $1 . \theta_{1}$ is a monomial over $\mathscr{D}$. Then

$$
\begin{aligned}
g= & P+\sum_{j=1}^{k_{1}} \frac{P_{1, j}}{\left(Q_{1}\right)^{j}}+a_{1} \log Q_{1} \\
& +\sum_{j=1}^{k_{2}} \frac{P_{2, j}}{\left(Q_{2}\right)^{j}}+a_{2} \log Q_{2} \\
& +\sum_{j=1}^{k_{r}} \frac{P_{r, j}}{\left(Q_{r}\right)^{j}}+a_{r} \log Q_{r}+\sum_{i=1}^{m} b_{i} \log h
\end{aligned}
$$


where $P, P_{i, j}, Q_{i}$ are in $\mathscr{D}\left[\theta_{1}\right] ; a_{i}, b_{i}$ els $K, h_{i}$ els $\mathscr{D}$; degree $P_{i j}<$ degree $Q_{i} ;$ the $Q_{i}$ are monic and irreducible.

We use the discussion preceding this theorem to conclude first that each $P_{i, j}=0$, for otherwise, using part (b) of that discussion, the right-hand side would have, after differentiation, a term $R /\left(Q_{i}\right)^{k_{i}+1}$. Thus $g=P+\sum_{j=1}^{r} a_{j} \log Q_{j}+\sum_{i=1}^{m} b_{i} \log h_{i}$. Now by part (c), each $a_{j}=0$; for otherwise the partial fraction decomposition of $f$ would have a term $S / Q_{j}$. Now if $\theta_{1}=\exp f_{1}$, then $P$ must be of degree 0 in $\theta_{1}$ and $g=P+\sum_{i=1}^{m} b_{i} \log h_{i}$ as required. If $\theta_{1}=\log f_{1}$, then $P=c \log f_{1}+v$ where $v \in \mathscr{D}$ and $c \in K$. Then $g=v+c \log f_{1}+\sum_{i=1}^{m} b_{i} \log h_{i}$ which is again in our desired form.

Case 2. $\theta_{1}$ is algebraic over $\mathscr{D}$.

$$
f=\left(w_{0}\right)^{\prime}+\sum_{i=1}^{k} d_{i} \frac{w_{i}^{\prime}}{w_{i}}
$$

Adding conjugates over $\mathscr{D}$ we get:

$$
m f=\left(\text { Trace } w_{0}\right)^{\prime}+\sum_{i=1}^{k} d_{i} \frac{\left(\text { Norm } w_{i}\right)^{\prime}}{\operatorname{Norm} w_{i}}
$$

where $m$ is a positive integer. Thus here too, $g$ is of the desired form and our proof is complete.

A question that arises is: Are any constants, not in $\mathscr{D}$, necessary for the integration of elements of $\mathscr{D}$ ? The example

$$
\int \frac{d z}{z^{2}-2}=\frac{\sqrt{ } 2}{4} \log \frac{z-\sqrt{ } 2}{z+\sqrt{ } 2}
$$

seems to indicate that one must adjoin $\sqrt{ } 2$ to $Q(z)$ in order to perform the integration. We now show this rigorously.

Proposition 1.1. Let $\mathscr{F}$ be elementary over $K(z)$, where $K$ is a field of constants and $z$ is transcendental over $K$ with $z^{\prime}=1$. Suppose $f \in \mathscr{F}$ and $f^{\prime}=1 /\left(z^{2}-2\right)$, then $\sqrt{ } 2 \in \mathscr{F}$.

Proof. Without loss of generality, we may assume that $K$ is the constant field of $\mathscr{F}$. Thus, by the first statement of Liouville's theorem, we get that $f=v_{0}+$ $\sum_{i=1}^{k} c_{i} \log v_{i}$ where $v_{0}, v_{i}$ are in $K(z)$ and the $c_{i}$ are in $K$. We may assume that the $v_{i}$, for $i>0$, are irreducible elements of $K[z]$. Note that if $\sqrt{ } 2 \notin K$, then $z^{2}-2$ is irreducible over $K$.

We decompose $v_{0}$ into partial fractions over $K$ and then differentiate the expression $v_{0}+\sum_{i=1}^{k} c_{i} \log v_{i}$. Now reasoning as in the proof of the first statement of Liouville's theorem, we see that $v_{0}$ must be in $K[z]$, for otherwise $1 /\left(z^{2}-2\right)$ would have to have an irreducible factor of degree greater than 1 in its denominator. Also, the only summand in the second term is $c \log \left(z^{2}-2\right)$. Furthermore, $v_{0}=a z$ $+b$. Thus, $f=a z+b+c \log \left(z^{2}-2\right), a, b$ and $c$ els $K$. Then $1 /\left(z^{2}-2\right)=a+2 c z /\left(z^{2}-2\right)$ which is impossible, by the uniqueness of a partial fraction decomposition. Thus $\sqrt{ } 2 \in \mathscr{F}$. 
It would seem that the new constants needed in integrating an element of $\mathscr{D}$ should all be algebraic over $K$. This is indeed the case.

Theorem of Liouville-Second Statement (Strong Liouville Theorem). Let $\mathscr{D}$ be a differential field with constant field $K$. Suppose $f \in \mathscr{D}$ and there is a $g$ elementary over $\mathscr{D}$ such that $g^{\prime}=f$. Then there are a $v_{0} \in \mathscr{D}, c_{i} \in \bar{K}(\bar{K}=$ algebraic closure of $K), v_{i} \in \bar{K} \mathscr{D}$ such that $f=v_{0}^{\prime}+\sum_{i=1}^{k} c_{i} v_{i}^{\prime} / v_{i}$ where every automorphism of $\bar{K} \mathscr{D}$ over $\mathscr{D}$ permutes the terms of the sum.

Lemma. (For the proof, see [3,p. 33].) Let $x_{1}, \ldots, x_{n}$ be indeterminates over $\mathscr{D}$, $p\left(x_{1}, \ldots, x_{n}\right)$ and $q\left(x_{1}, \ldots, x_{n}\right)$ are in $\mathscr{D}\left[x_{1}, \ldots, x_{n}\right]$. Suppose that there are $c_{1}, \ldots, c_{n}$ in $C(=$ constant field of a universal extension of $\mathscr{D})$ such that $p\left(c_{1}, \ldots, c_{n}\right)=0$ and $q_{1}\left(c_{1}, \ldots, c_{n}\right) \neq 0$. Then there are $k_{1}, \ldots, k_{n}$ in $\bar{K}$ such that $p\left(k_{1}, \ldots, k_{n}\right)=0$ while $q\left(k_{1}, \ldots, k_{n}\right) \neq 0$.

Proof of second statement. By adjoining constants $c_{1}, \ldots, c_{n}$ in $C$ to $\mathscr{D}$ we can apply the First Statement to the present situation with $\mathscr{D}\left(c_{1}, \ldots, c_{n}\right)$ replacing $\mathscr{D}$. Thus we have that

$$
g=\frac{p_{0}\left(c_{1}, \ldots, c_{n}\right)}{q_{0}\left(c_{1}, \ldots, c_{n}\right)}+\sum_{i=1}^{k} c_{i} \log \frac{p_{i}\left(c_{1}, \ldots, c_{n}\right)}{q_{i}\left(c_{1}, \ldots, c_{n}\right)},
$$

where each $p_{i}, q_{i}$ is a polynomial with coefficients in $\mathscr{D}$.

$$
\begin{aligned}
p_{i}\left(c_{1}, \ldots, c_{n}\right) \neq 0, & i=1, \ldots, k, \\
q_{i}\left(c_{1}, \ldots, c_{n}\right) \neq 0, & i=0, \ldots, k .
\end{aligned}
$$

By differentiating, we get

$$
f=\frac{\bar{p}_{0}^{\prime} \bar{q}_{0}-\bar{q}_{0}^{\prime} \bar{p}_{0}}{\bar{q}_{0}^{2}}+\sum_{i=1}^{k} c_{i} \frac{\bar{p}_{i}^{\prime} \bar{q}_{i}-\bar{q}_{i}^{\prime} \bar{p}_{i}}{\bar{p}_{i} \bar{q}_{i}}
$$

where $\bar{p}_{i}$ and $\bar{q}_{i}$ are $p_{i}$ and $q_{i}$ evaluated at $\left(c_{1}, \ldots, c_{n}\right)$.

Thus, $c_{1}, \ldots, c_{n}$ satisfy the polynomial conditions $P\left(x_{1}, \ldots, x_{n}\right)=0$ and

$$
Q\left(x_{1}, \ldots, x_{n}\right)=q_{0}^{2}\left(x_{1}, \ldots, x_{n}\right) \prod_{1}^{k} p_{i}\left(x_{1}, \ldots, x_{n}\right) q_{i}\left(x_{1}, \ldots, x_{n}\right) \neq 0,
$$

$P$ being obtained from $\left(^{*}\right)$ by a simple transformation. Thus, by the lemma, $P\left(k_{1}, \ldots, k_{n}\right)=0$ and $Q\left(k_{1}, \ldots, k_{n}\right) \neq 0$ where $\left(k_{1}, \ldots, k_{n}\right) \in \bar{K}^{n}$.

By backtracking from these conditions we get $f=\bar{v}_{0}^{\prime}+\sum_{i=1}^{k} k_{i} \bar{v}_{i}^{\prime} / \bar{v}_{i}$ where $\bar{v}_{i}$ are els of $\bar{K} \mathscr{D}$, viz. $\bar{v}_{i}=p_{i}\left(k_{1}, \ldots, k_{n}\right) / q_{\imath}\left(k_{1}, \ldots, k_{n}\right), i=0, \ldots, k$.

Taking traces we get $l f=\left(\text { Trace } \bar{v}_{0}\right)^{\prime}+\sum_{i=1}^{k}$ Trace $k_{i} \bar{v}_{i}^{\prime} / \bar{v}_{i}, l$ a positive integer. Taking $v_{0}=(1 / l)$ Trace $\bar{v}_{0}$, we get our desired result.

Open Problem. In the preceding theorem, if $g \in \mathscr{F}$, where $\mathscr{F}$ is elementary over $\mathscr{D}$, can $v_{0}, v_{i}, k_{i}$, and $\log v_{i}, i=1, \ldots, k$, be chosen so as also to be in $\mathscr{F}$ ? 
Proposition 1.2. Let $\mathscr{D}$ be a differential field, $f \in \mathscr{D}$.

(a) Suppose $f \neq 0$ and $\theta=\log f$ is not a monomial over $\mathscr{D}$. Then $\theta=g+c, g \in \mathscr{D}$, $c \in C$.

(b) Suppose $\theta=e^{f}$ is not a monomial over $\mathscr{D}$. Then $\theta^{n}=d h$ where $n \in Z, d \in C$, and $h \in \mathscr{D}$.

Proof. (a) $f^{\prime} \mid f=\theta^{\prime}$ where $\theta$ is algebraic over $\mathscr{D}(k), k \in C$.

Then after adding conjugates, using the argument of the Strong Liouville Theorem which is based on the lemma and adding conjugates again we get that $f^{\prime} \mid f=g^{\prime}$ for $g \in \mathscr{D}$. Thus $\theta=g+c, c \in C$.

(b) Here $\theta^{\prime} \mid \theta=f^{\prime}$ with $\theta$ algebraic over $\mathscr{D}(k), k \in C$.

Reasoning as in (a) we get that $h^{\prime} / h=n f^{\prime}$, where $n \in Z$ and $h \in \mathscr{D}$. Thus $\theta^{n}=d h$, $h \in C$.

The following easy consequence of 1.2 will be useful to us in formulating the integration problem.

Proposition 1.3. Let $\theta$ be a monomial over $\mathscr{D} . \theta=\exp f$ or $\theta=\log f, f \in \mathscr{D}$. Suppose $\psi=\exp f$ or $\psi=\log f$ (i.e., $\psi$ satisfies the same defining equation as $\theta$ ), then $\psi$ is also a monomial over $\mathscr{D}$ and $\mathscr{D}(\theta)$ is differentially isomorphic to $\mathscr{D}(\psi)$ with $\theta \rightarrow \psi$.

In the example on page 168 where $\theta=z$ and $\psi=z+2 \pi i, \mathscr{D}(\theta)=Q\left(z, e^{z}\right)$ while $\mathscr{D}(\psi)=Q\left(2 \pi i, z, e^{z}\right)$ and these fields are not isomorphic.

Proposition 1.4. Let $\mathscr{D}$ be a differential field, $f \in \mathscr{D}$. Suppose that there is a $g$, elementary over $\mathscr{D}$ such that $g^{\prime}=f$. Then there is an $h$, regular elementary over $\mathscr{D}$ such that $h^{\prime}=f$.

Proof. By the Strong Liouville Theorem, there is a $j$ in $\mathscr{D}\left(\sigma, \log v_{1}, \ldots, \log v_{k}\right)$, where $\sigma$ is algebraic over the constant field of $\mathscr{D}$ and $v_{1}, \ldots, v_{k}$ are in $\mathscr{D}(\sigma)$, such that $j=v_{0}+\sum_{i=1}^{k} c_{i} \log v_{i}, v_{0} \in \mathscr{D}, c_{i} \in \mathscr{D}(\sigma)$, and $j^{\prime}=f$. By 1.2 , each $\log v_{i}$ differs by an additive constant from an element of $\mathscr{D}\left(\sigma, \log v_{1}, \ldots, \log v_{i-1}\right)$ or else is a monomial over the latter field. Thus, by subtracting a constant from $j$, we get an $h$, regular elementary over $\mathscr{D}$, such that $h^{\prime}=f$.

2. Formulation of the problem of integration in finite terms. We begin with a few remarks on recursive functions. For complete details, see [1].

A function on and to the natural numbers is called recursive iff there is an algorithm for computing $f(n)$ for each $n$. There are also precise mathematical definitions of recursive functions which have all been shown to be equivalent to each other. The hypothesis that they all are equivalent to the definition we have given is called "Church's thesis."

A set of natural numbers is recursively enumerable iff it is the range of a recursive function. If a set of numbers has a recursive characteristic function, then the set is called recursive. This means that it and its complement are recursively enumerable 
or that there is an algorithm for deciding whether or not a given natural number is in the set.

Suppose there is a one to one correspondence between a countable set of mutually distinguishable objects $\mathscr{T}$ and the natural numbers such that given an $\alpha$ in $\mathscr{T}$ one can compute the associated natural number, and vice versa. Then $\mathscr{T}$ is said to have been given a Gödel numbering. We define recursively enumerable and recursive subsets of $\mathscr{T}$ as sets whose associated Gödel numbers form a recursively enumerable or recursive set of natural numbers.

The $\mathscr{T}$ we will deal with will be our symbols for the elementary functions. We will describe a language fragment which comes very close to the way a mathematician customarily denotes these functions. Our functions lie in differential fields of meromorphic functions on a region of the complex plane or a Riemann surface which are finitely generated over the rational numbers, e.g.,

$$
\mathscr{D}=Q\left(a_{1}, \ldots, a_{n}, b, z, \exp z^{2}, \log \left(z+\exp z^{2}\right), \sqrt{ }\left(\log \left(z+\exp z^{2}\right)\right)\right)
$$

where $a_{1}, \ldots, a_{n}$ are constants, algebraically independent over $Q, b$ is algebraic over $Q\left(a_{1}, \ldots, a_{n}\right), z$ is the identity function, etc. We can represent all members of $\mathscr{D}$ if we have one symbol for each of the generators of $\mathscr{D}$ and binary operation symbols for $+, \cdot,-$, and $\div$.

It is easy enough to invent symbols for logarithms and exponentials of functions already described-just write log or exp in front of the previously defined symbol. However, algebraic operations cause a problem. Not all of them can be expressed by means of radical signs. Thus, we have invented " $\gamma$ symbols" to denote them.

We consider sequences of symbols:

$$
e=\left\langle 1_{e}, \pi_{e}, \alpha_{1}, \ldots, \alpha_{k}, \gamma\left[\left(\left(\left(\gamma^{l}+{ }_{e} \beta_{1} \cdot{ }_{e} \gamma^{l-1}\right)+{ }_{e} \cdots+{ }_{e} \beta_{l}\right)\right)\right], z_{e}, \delta_{1}, \ldots, \delta_{m}\right\rangle
$$

( $k, m \geqq 0, l>1, \pi_{e}$ and the symbol following $\alpha_{k}$ possibly missing), which we call elementary field descriptions (efd's). Along with $e$, we will consider the nested sequence: $\Omega_{1_{e}}, \Omega_{\pi_{e}}, \Omega_{\alpha_{1}}, \ldots, \Omega_{\delta_{m}}=\Omega_{e}$. An $\Omega_{\sigma}$ is the smallest set of terms containing $1_{e}, \ldots, \sigma$ and closed under the binary operation symbols $+_{e},-{ }_{e}, \cdot_{e}, \div_{e}$.

We divide the symbols of $e$ into two sorts-the $\gamma$ symbols and the non- $\gamma$ symbols. A $\gamma$ symbol is of the form: $\gamma\left[\left(\left(\left(\gamma^{n}+{ }_{e} \lambda_{1} \cdot{ }_{e} \gamma^{n-1}\right)+{ }_{e} \cdots+{ }_{e} \lambda_{n}\right)\right)\right]$ where $n>1, \gamma^{j}$ means $\left(\left(\left(\gamma \cdot e_{e} \gamma\right) \cdot{ }_{e} \gamma \cdots\right)\right) j$ times, $j \geqq 1$, and the $\lambda_{i}$ are in $\Omega_{\sigma}$, where $\sigma$ is the symbol directly preceding, in the sequence $e$, the $\gamma$ symbol we are discussing. Besides the $\gamma$ symbol explicitly indicated in the $e$ above, some of the $\delta_{i}$ may be $\gamma$ symbols. However, $1_{e}, \pi_{e}, \alpha_{1}, \ldots, \alpha_{k}$ and $z_{e}$ are non- $\gamma$ symbols.

Each $\delta_{j}$ satisfies one of the following three conditions:

(1) $\delta_{j}$ is a $\gamma$ symbol.

(2) There is a $\zeta$ in $\Omega_{\delta_{j-1}}\left(\Omega_{\delta_{0}}=\Omega_{z_{e}}\right)$, such that $\delta_{j}$ is the symbol exp $\exp _{e}$.

(3) There is a $\zeta$ in $\Omega_{\delta_{j-1}}$, such that $\delta_{j}$ is the symbol $\log _{e} \zeta$.

Definition. We call $\left(\mathscr{D}_{A}, V\right)$ an e-model, where $\mathscr{D}_{A}$ is a differential field of 
meromorphic functions on a region $A$ and $V$ is a mapping whose domain is a subset $\Phi(e, V)$ of $\Omega_{e}$ and whose range is $\mathscr{D}_{A}$, iff

(1) $V\left(1_{e}\right)=1$.

(2) $V\left(\pi_{e}\right), V\left(\alpha_{1}\right), \ldots, V\left(\alpha_{k}\right)$ are complex numbers which are algebraically independent over $Q$ with $V\left(\pi_{e}\right)=\pi$.

(3) For the $\gamma$ symbol following $\alpha_{k}$ we have $V(\gamma[-])$ is a complex number and $V(\gamma[-])^{l}+V\left(\beta_{1}\right) V(\gamma[-])^{l-1}+\cdots+V\left(\beta_{l}\right)=0$ and this is the monic irreducible equation satisfied by $V(\gamma[-])$ over $Q\left(\pi, V\left(\alpha_{1}\right), \ldots, V\left(\alpha_{k}\right)\right)$.

(4) $V\left(z_{e}\right)=I$ where $I$ is the identity function on $A$.

(5) If $\delta_{j}=\gamma\left[\left(\left(\left(\gamma^{n}+{ }_{e} \lambda_{1} \cdot{ }_{e} \gamma^{n-1}\right)+{ }_{e} \cdots+{ }_{e} \lambda_{n}\right)\right)\right]$, then $V\left(\delta_{j}\right)^{n}+V\left(\lambda_{1}\right) V\left(\delta_{j}\right)^{n-1}+\cdots$ $+V\left(\lambda_{n}\right)=0$.

(6) If $\delta_{j}=\exp _{e} \zeta$ then $V\left(\delta_{j}\right)=e^{V(\zeta)}$, where $e$ is the exponential function.

(7) If $\delta_{j}=\log _{e} \zeta$ then $V(\zeta)=e^{V\left(\delta_{j}\right)}$.

(8) If $\alpha, \beta$ are in $\Phi(e, V)$ then so are $\alpha+{ }_{e} \beta,-{ }_{e} \alpha$ and $\alpha \cdot{ }_{e} \beta$. If furthermore $V(\beta) \neq 0$ then $\alpha \div{ }_{e} \beta$ is also in $\Phi(e, V)$ and we have

$$
\begin{aligned}
V\left(\alpha+{ }_{e} \beta\right) & =V(\alpha)+V(\beta), \quad V\left(-{ }_{e} \alpha\right)=-V(\alpha), \\
V\left(\alpha \cdot{ }_{e} \beta\right) & =V(\alpha) V(\beta), \quad V\left(\alpha \div{ }_{e} \beta\right)=V(\alpha) / V(\beta) .
\end{aligned}
$$

It is clear that $\mathscr{D}=Q\left(\pi, V\left(\alpha_{1}\right), \ldots, V\left(\alpha_{k}\right), V(\gamma[-]), I, V\left(\delta_{1}\right), \ldots, V\left(\delta_{m}\right)\right)$. The only reason we use fields of meromorphic functions in defining an $e$ model instead of abstract differential fields is because of the properties used in Proposition 2.2. In the meromorphic case $\exp _{e} \zeta$ does not refer to any solution of $\theta^{\prime}-\zeta^{\prime} \theta=0$ but a special one. Similarly, $\log _{e} \zeta$ refers to a member of a certain countable set of solutions of $\zeta^{\prime}-\theta^{\prime} \zeta=0$.

We note that while, on a region $A$ of the complex plane, there is an uncountable number of elementary functions, we have invented only a countable number of symbols to denote them. The paradox is explained by the fact that an efd may have an uncountable number of $e$ models, all on the same region. E.g., $\left\langle 1_{e}, \alpha_{1}, \ldots, \alpha_{k}, z_{e}\right\rangle$ has $c$ distinct $e$ models which are obtained by letting $\alpha_{1}, \ldots, \alpha_{k}$ vary over all sets of $k$ algebraically independent elements of $\mathbf{C}$. All these $e$ models are differentially isomorphic. For our purposes, we may treat them as identical. For example, $\int e^{\pi z} \log (\sqrt{ } \pi z) d z$ and $\int e^{e z} \log (\sqrt{ } e z) d z$ are either both elementary or both nonelementary.

Definition. We say that an efd $e$ is regular if there is an $e$ model $\left(\mathscr{D}_{A}, V\right)$ such that:

(1) For each $\delta_{i}$ of the form $\gamma\left[\left(\left(\left(\gamma^{n}+{ }_{e} \lambda_{1} \cdot \gamma^{n-1}\right)+{ }_{e} \cdots+{ }_{e} \lambda_{n}\right)\right)\right]$,

$$
V\left(\delta_{i}\right)^{n}+V\left(\lambda_{1}\right) V\left(\delta_{i}\right)^{n-1}+\cdots+V\left(\lambda_{n}\right)=0
$$

is the monic irreducible equation satisfied by $V\left(\delta_{i}\right)$ over $Q\left(\pi, V\left(\alpha_{1}\right), \ldots, V\left(\delta_{i-1}\right)\right)$.

(2) For each $\delta_{i}$ of the form $\exp _{e} \zeta$ or $\log _{e} \zeta, V\left(\delta_{i}\right)$ is a monomial over

$$
Q\left(\pi, V\left(\alpha_{1}\right), \ldots, V\left(\delta_{i-1}\right)\right) \text {. }
$$

Thus, $\mathscr{D}_{A}$ is regular elementary over $Q\left(\pi, V\left(\alpha_{1}\right), \ldots, I\right)$. 
Proposition 2.1. Let $\mathscr{D}$ be (regular) elementary over $K(z)$ where $K$ is a subfield of the complex numbers which is finitely generated over the rationals and $z$ is a solution of $z^{\prime}=1$. For any region $B$ of the complex plane, there is a subregion $A$, a field $\mathscr{D}_{A}$ of functions meromorphic on $A$ which is differentially isomorphic to $\mathscr{D}$, a (regular) efd $e$, and a mapping $V$ such that $\left(\mathscr{D}_{A}, V\right)$ is an e model.

Proof. This can be easily derived from some well-known results in elementary complex analysis. The existence of $\mathscr{D}_{A}$ is also a consequence of Seidenberg's theorem that any differential field that is finitely generated over the rationals is differentially isomorphic to a field of meromorphic functions. See [12].

Definition. Let $e$ be an efd. Then $\Sigma_{e}=\left\{\alpha \in \Omega_{e}: \exists\right.$ an $e$ model $\left(\mathscr{D}_{A}, V\right)$ and an $f$, regular elementary over $\mathscr{D}_{A}$ such that $\alpha \in \Phi(e, V)$ and $\left.V(\alpha)=(d / d z) f\right\}$.

Note that if we substitute, in the above definition, "elementary" for "regular elementary" the new set is the same as the old (Proposition 1.4). Proposition 2.1 tells us that $f$ can be realized as a meromorphic function on some subregion of $A$.

The Problem of Integration in Finite Terms. Is $\Sigma_{e}$ a recursive subset of $\Omega_{e}$ ?

This problem can be intuitively stated as: Given an arbitrary symbol for an elementary function, is there some way of choosing the branches for the logarithmic and algebraic operations involved so that the resulting function exists and has an elementary indefinite integral? For example, the answer for $\int\left(\log e^{z}-z\right) \exp z^{2} d z$ is yes since we can choose $\log e^{z}=z$. Note that the efd

$$
\left\langle 1_{e}, z_{e}, \exp _{e} z_{e}, \log _{e} \exp _{e} z_{e}, \exp _{e}\left(z_{e} \cdot{ }_{e} z_{e}\right)\right\rangle
$$

is not regular. Cf. the example on page 172.

PROPOSITION 2.2. There is an e such that $\Sigma_{e}$ is not a recursive subset of $\Omega_{e}$, i.e., for which the above problem is undecidable.

Proof. It follows from [2, p. 430] and [1, p. 103] that there is a polynomial $P\left(w, x_{1}, \ldots, x_{k}, y_{1}, \ldots, y_{k}\right)$ with integer coefficients such that the set of natural numbers $S$, defined below is not recursive.

$$
\begin{gathered}
s \in S \leftrightarrow \text { integers } X_{1}, \ldots, X_{k} \text { such that } P\left(s, X_{1}, \ldots, X_{k}, 2^{X_{1}}, \ldots, 2^{X_{k}}\right) \\
=G\left(s, X_{1}, \ldots, X_{k}\right)=0 .
\end{gathered}
$$

Let $F_{j}=(1 / 2 \pi i)\left(\log \exp z^{j}-z^{j}\right), j=1, \ldots, k$. It is clear that for each $s, H(s)$ $=G\left(s, F_{1}, \ldots, F_{k}\right)$ is a symbol for an elementary function and that $\int H(s) \exp z^{2} d z$ is elementary for some choice of the branches of the logarithms iff $s \in S$.

Thus, for any $e$ such that $\Omega_{e}$ contains a symbol $H(s) \exp z^{2}, \Sigma_{e}$ is not a recursive subset of $\Omega_{e}$.

It would be nice to have a proof of the above proposition without having $\pi$ play its special role.

We will get around the difficulties indicated in Proposition 2.2 by choosing our symbols so that no matter what branches of functions, which are built up using 
logarithmic or algebraic operations, are chosen or what region $A$ we use, in defining our $e$ model, the integrability of a function represented by a symbol depends only on the symbol. This is achieved by restricting ourselves to regular efd's. The next proposition which can be derived from 1.3 summarizes the properties of these efd's.

Proposition 2.3. Let e be a regular efd, $\left(\mathscr{D}_{A}, V_{A}\right)$ and $\left(\mathscr{D}_{B}, V_{B}\right)$ e models. Then $\Phi\left(e, V_{A}\right)=\Phi\left(e, V_{B}\right)$ and we now write this set as $\Phi_{e}$. The correspondence $V_{1}(\alpha)$ $\leftrightarrow V_{2}(\alpha)$, for $\alpha \in \Phi_{e}$, is a differential isomorphism of $\mathscr{D}_{A}$ and $\mathscr{D}_{B}$. Let $\Sigma_{e}\left(\mathscr{D}_{A}, V_{A, e}\right)$ $=\left\{\alpha \in \Phi_{e}: \exists\right.$ an $f$ which is regular elementary over $\mathscr{D}_{A}$ such that $\left.(d / d z) f=V_{A, e}(\alpha)\right\}$. Then $\Sigma_{e}\left(\mathscr{D}_{A}, V_{A, e}\right)=\Sigma_{e}\left(\mathscr{D}_{B}, V_{B, e}\right)=\Sigma_{e}$.

Also note that for a regular efd $e, \Phi_{e}$ is a recursive subset of $\Omega_{e}$ and the relation $\equiv_{e}$, defined by $\alpha \equiv_{e} \beta$ if $V(\alpha)=V(\beta)$ for some $e$ model $\left(\mathscr{D}_{A}, V\right)$ is well defined and recursive. Thus we can tell whether or not two elementary functions are equal when they are both in a common model of a regular efd.

For a regular efd $e, \Sigma_{e}$ is a recursively enumerable subset of $\Phi_{e}$. This follows from the Strong Liouville Theorem.

It should be pointed out that if $e$ is regular and has $e$ models $\mathscr{D}_{A}$ and $\mathscr{D}_{B}$, then it is not necessarily true that the functions of $\mathscr{D}_{A}$ can be continued analytically into the corresponding functions of $\mathscr{D}_{B}$. E.g., $\log x^{2}$ represents two distinct monogenic analytic functions. Thus, the existence of the differential isomorphism of Proposition 2.3 could not be proved by analytic continuation.

3. Integration of elements of pure monomial extensions. We saw, in $\$ 2$, how to rigorously formulate the problem of integration in finite terms. We intend now to solve a portion of it with the theorems:

(1) Let $\mathscr{E}$ be the set of those elementary field descriptions $e$ in which each $\delta_{i}$ is either of the form $\exp _{e} \zeta$ or $\log _{e} \eta$ (i.e., no $\delta_{i}$ is a $\gamma$ symbol). Then the subset of regular efd's of $\mathscr{E}$ is recursive.

(2) Let e be a regular efd of $\mathscr{E}$, then $\Sigma_{e}$ is recursive.

Roughly, this means that we can handle any elementary function which involves just the rational operations, exponentials and logarithms, providing these latter two cannot be replaced by adjoining constants and performing algebraic operations. E.g., we can look at

$$
\int \exp \left(\frac{e^{z}}{z}-\frac{\log z}{z^{2}}\right) \log z d z
$$

but not at $\int \exp \left(1+\frac{1}{2} \log \left(z^{3}-1\right)\right) d z$ with our algorithm (as it is given here).

In his book [8], Mordoukhay-Boltovskoy makes a pretense of showing that "given an integral of a rational transcendental function, then it is always possible, by means of a finite number of algebraic operations, to express it in a finite number of terms or assert its inexpressibility in finite terms" [8, pp. 244-245]. What he means is our theorem (2) above.

Using the concepts and terminology which we have developed, his method is as 
follows: Suppose we are given a function $P(\theta) / Q(\theta)$ where $P(\theta), Q(\theta)$ are els of $\mathscr{D}[\theta], \mathscr{D}=K\left(z, \theta_{1}, \ldots, \theta_{n-1}\right), \theta=\theta_{n}, \theta_{i}$ a monomial over $K\left(z, \theta_{1}, \ldots, \theta_{i-1}\right)$. One can show that if elementary,

$$
\begin{aligned}
\int \frac{P(\theta)}{Q(\theta)} & =\int \frac{P(\theta)}{\left(\theta-\alpha_{1}\right)^{k_{1}} \cdots\left(\theta-\alpha_{n}\right)^{k_{n}}} \\
& =\frac{R(\theta)}{\left(\theta-\alpha_{1}\right)^{k_{1}-1} \cdots\left(\theta-\alpha_{n}\right)^{k_{n}-1}}+\sum_{i=1}^{m} c_{i} \log \left(\theta-\alpha_{i}\right)+S,
\end{aligned}
$$

where one factors the denominator of $Q$ over the algebraic closure of $\mathscr{D}, R(\theta)$ $\in \mathscr{D}[\theta], c_{i} \in \bar{K}$, and $S=\Sigma d_{i} \log \delta_{i}, d_{i} \in \bar{K}, \delta_{i} \in \bar{K} \mathscr{D}$. After differentiating and clearing denominators, one gets $A R^{\prime}+B R+\sum_{i=1}^{m} c_{i} C_{i}+S^{\prime} D=0$, where $A, B, C_{i}, D$ are known polynomials in $\theta$, the $c_{i}$ are unknown constants and $S^{\prime}$ is an unknown element of $\mathscr{D}$. A bound $k$ can be found for the degree of the polynomial $R$. Thus, substituting $R=a_{k} \theta^{k}+\cdots+a_{0}$ into the preceding equation, one gets a simultaneous system of first order linear differential equations for the $a_{i}$ 's. These equations have the parameters $c_{i}$ and $S^{\prime}$. One must determine if these equations have a solution $a_{0}, \ldots, a_{k}$ with $a_{i} \in \mathscr{D}, c_{i} \in \bar{K}, S^{\prime} \in \mathscr{D}$.

At this point, Mordoukhay-Boltovskoy waves his hand. (This is not to imply that he is clear and convincing in getting this far.) He refers to Liouville [6] and Sintzov [13] for methods of solving linear systems. However, these writers only discuss the problem when the coefficients are rational functions of $z$, and the results do not generalize in any obvious way. Besides, Sintzov's method of solution ( $\$ 23$, beginning on p. 146 of the August issue) is erroneous. We will show that the testing of the systems, which arise in the integration of elements of $\mathscr{D}(\theta)$, for solutions in their coefficient field can always be reduced to the testing of a succession of single first order linear equations. Mordoukhay-Boltovskoy does not seem to have been aware of this important fact. For example, in [8, p. 160] he reduces a system of two first order equations to a second order equation.

Also very troublesome in this method are the parameters $c_{i}$ and $S^{\prime}$-especially the $S^{\prime}$. It is not clear how to handle them. In the trivial examples MordoukhayBoltovskoy works out, no difficulties arise. But there is no certainty that this will always be so. The problem of handling $S^{\prime}$ becomes manageable if we first decompose both our integrand and the functions occurring in the integral into partial fractions over $\mathscr{D}$ and then find the conditions on these unknown functions. This idea is found for the logarithmic case in Ostrowski's paper [9].

We will not be as formal as we were in \$2-e.g., we will usually not make a distinction between the terms of a language and the functions they represent. Also, we will not use the language of recursive function theory. In $\S 3$, we work exclusively with fields $K\left(z, \theta_{1}, \ldots, \theta_{n}\right), K$ a field of constants which is finitely generated over $Q$, $z$ a solution of $z^{\prime}=1$ and each $\theta_{i}$ a monomial over $K\left(z, \theta_{1}, \ldots, \theta_{i-1}\right)$. Proposition 2.1 tells us that we have a regular efd for this field and so are able to decide if two elements of it are equal. 
We first dispose of the problem of factoring a polynomial in $\theta_{n}$ over

$$
\bar{K}\left(z, \theta_{1}, \ldots, \theta_{n-1}\right),
$$

$\bar{K}=$ alg. closure of $K$.

Problem. Let $K$ be finitely generated over $Q$. Let $x_{1}, \ldots, x_{n}$ be algebraically independent over $K$. To factor a polynomial $P\left(x_{1}, \ldots, x_{n}\right) \in K\left[x_{1}, \ldots, x_{n}\right]$ into irreducible factors $q_{1}, \ldots, q_{k}$ over $\bar{K}$. To find an equation, $p(b)=0$, which is minimal for $b$ over $K$, such that $q_{1}, \ldots, q_{k}$ are in $K(b)\left[x_{1}, \ldots, x_{n}\right]$.

Solution. We refer to results proved on field theoretic algorithms in $[5, \S 19]$ and $[14, \S 42]$.

By the Kronecker trick [14, p. 135], we transform $P\left(x_{1}, \ldots, x_{n}\right)$ into $P^{*}(t)$, a polynomial in a single indeterminate with coefficients in $K$. Since $K$ is finitely generated over $Q$, we can factor a polynomial over it in the manner described in [14]. Thus, we can factor $P^{*}(t)$ over $K$ to see if it has multiple factors or not. If it has, we replace it by a polynomial without multiple factors, $\bar{P}^{*}(t)$, having the same irreducible factors as $P^{*}(t)$. Next we find a primitive element $b$ for the splitting field of $\bar{P}^{*}(t)$ over $K$ [5, p. 66, Satz 1]. Then we factor $P^{*}(t)$ over $K(b)[5$, p. 68 , Satz 3], or $\left[14\right.$, p. 136]. Then we convert back to a factorization of $P\left(x_{1}, \ldots, x_{n}\right)$ using the Kronecker device.

COROllary. We may factor a polynomial $P\left(x_{n}\right) \in K\left(x_{1}, \ldots, x_{n-1}\right)\left[x_{n}\right]$ over $\bar{K}\left(x_{1}, \ldots, x_{n-1}\right)$.

In our integration algorithm, the polynomial $P\left(\theta_{n}\right)$ to be factored over

$$
\bar{K}\left(z, \theta_{1}, \ldots, \theta_{n-1}\right)
$$

is a factor, irreducible over $K\left(z, \theta_{1}, \ldots, \theta_{n-1}\right)$, of the denominator of the

$$
M\left(\theta_{n}\right) / N\left(\theta_{n}\right) \in K\left(z, \theta_{1}, \ldots, \theta_{n}\right)
$$

which we wish to integrate. To use the language of $\S 2$, if we start with a regular efd $e=\left\langle 1_{e}, \alpha_{1}, \ldots, \alpha_{k}, \gamma[-], z_{e}, \delta_{1}, \ldots, \delta_{n}\right\rangle$ which describes $K\left(z, \theta_{1}, \ldots, \theta_{n}\right)$ $\left(V\left(\delta_{i}\right)=\theta_{i}\right)$, we now work with the sequence $e^{\prime}=\left\langle 1_{e}, \ldots, \delta_{n}, \gamma_{b_{1}}[-]\right\rangle$ where

$$
V\left(\gamma_{b_{1}}[-]\right)=b_{1}
$$

is the primitive element obtained according to the preceding discussion for $P$. In the course of applying the algorithm to a particular function, we may make further extensions to our original efd $e$ by $\gamma$ symbols $\gamma_{b_{2}}[-], \ldots, \gamma_{b_{k}}[-]$ in order to obtain symbols for the factorization of other polynomials which come up.

LEMMA. Let $P_{1}=0, \ldots, P_{m}=0$ be a set of simultaneous linear algebraic equations with coefficients in $K\left(z, \theta_{1}, \ldots, \theta_{n}\right)$. Then one can find, in a finite number of steps, a system $\mathscr{S}$ of simultaneous linear equations with coefficients in $K$ such that $a\left(k_{1}, \ldots, k_{r}\right)$ $\in K^{r}$ satisfies $P_{1}=0, \ldots, P_{m}=0$ if $\left(k_{1}, \ldots, k_{r}\right)$ satisfies $\mathscr{S}$. 
Proof. It suffices to show this for a single equation $P=0$.

For $n=0$ our field is $K(z)$. Then $P=\left(R_{1}(z) / S_{1}(z)\right) x_{1}+\cdots+\left(R_{r}(z) / S_{r}(z)\right) x_{r}$, $S_{i}, R_{i}$, els $K(z) .\left(k_{1}, \ldots, k_{r}\right)$ satisfies $P$ iff $\left(k_{1}, \ldots, k_{r}\right)$ satisfies $R_{1}(z) \prod_{i \neq 1} S_{i}(z) x_{1}+\cdots$ $+R_{r}(z) \prod_{i \neq r} S_{i}(z) x_{r}$.

By collecting like powers of $z$ we get our desired system $\mathscr{S}$.

Assume the result is true for $n-1$. Then

$$
P=\left(R_{1}\left(\theta_{n}\right) / S_{1}\left(\theta_{n}\right)\right) x_{1}+\cdots+\left(R_{r}\left(\theta_{n}\right) / S_{r}\left(\theta_{n}\right)\right) x_{r} .
$$

We clear denominators and collect coefficients of like powers of $\theta_{n}$. We get a set of equations $Q_{1}=0, \ldots, Q_{m}=0$ with coefficients in $K\left(z, \theta_{1}, \ldots, \theta_{n-1}\right)$ which are satisfied by a $\left(k_{1}, \ldots, k_{r}\right)$ in $K^{r}$ iff $\left(k_{1}, \ldots, k_{r}\right)$ satisfies $P$. By the induction assumption, we replace each $Q_{i}$ by its equivalent system $\mathscr{S}_{i}$. Then $\mathscr{S}_{=} \bigcup_{i=1}^{m} \mathscr{S}_{i}$ is our desired system.

We can now state the

MAIN Theorem. Let $\mathscr{F}=K\left(z, \theta_{1}, \ldots, \theta_{n}\right), K$ is a field of constants which is finitely generated over $Q, z$ is transcendental over $K$ and a solution of $z^{\prime}=1$, each $\theta_{i} a$ monomial over $K\left(z, \theta_{1}, \ldots, \theta_{i-1}\right)$.

(a) Let $f \in \mathscr{F}$. Then one can determine in a finite number of steps whether there are $v_{0} \in \mathscr{F}, v_{i}$ els of $\bar{K} \mathscr{F}, i=1, \ldots, m$ and $c_{1}, \ldots, c_{m}$ in $\bar{K}$ such that $f=\left[v_{0}+\sum_{i=1}^{m} c_{i} \log v_{i}\right]^{\prime}$. If they do exist we can find them.

(b) Let $f, g_{i} i=1, \ldots, m$ be els of $\mathscr{F}$. Then one can find, in a finite number of steps, $h_{1}, \ldots, h_{r}$ in $\mathscr{F}$ and a simultaneous set $\mathscr{S}$ of linear algebraic equations in $m+r$ variables, with coefficients in $K$, such that $y^{\prime}+f y=\sum_{i=1}^{m} c_{i} g_{i}$ holds for $y \in \mathscr{F}$ and $c_{i}$ els of $K$ iff $y=\sum_{i=1}^{r} y_{i} h_{i}$ where $y_{i}$ els $K$ and $c_{1}, \ldots, c_{m}, y_{1}, \ldots, y_{r}$ satisfy $\mathscr{S}$.

Part (b), while not of particular interest in itself, serves to facilitate the proof, which is by induction on $n$ :

$$
n=0 \quad \mathscr{F}=K(z) .
$$

(a) Using the Strong Liouville Theorem, we may write, if $\int f$ (i.e., a $g$ such that $g^{\prime}=f$ ) is elementary:

$$
f=\left\{\begin{array}{c}
A_{k} z^{k}+\cdots+A_{0} \\
+\frac{A_{1, k_{1}}}{p_{1}^{k_{1}}}+\cdots+\frac{A_{1,1}}{p_{1}} \\
\vdots \\
+\frac{A_{s, k_{s}}}{p_{s}^{k_{s}}}+\cdots+\frac{A_{s, k_{s}}}{p_{s}}
\end{array}\right\}=\left\{\begin{array}{c}
B_{k+1} z^{k+1}+\cdots+B_{1} z+B_{0} \\
+\frac{B_{1, k_{1}-1}}{p_{1}^{k_{1}-1}}+\cdots+\frac{B_{1,1}}{p_{1}}+\int \frac{B_{1,0}}{p_{1}} \\
\vdots \\
+\frac{B_{s, k_{s}-1}}{p_{s}^{k_{s}-1}}+\cdots+\frac{B_{s, 1}}{p_{s}}+\int \frac{B_{s, 0}}{p_{s}}
\end{array}\right\}
$$

where the $A_{i}$ 's and $B_{i}$ 's are in $K$ : the $A_{i j}, B_{i, j}$ and $p_{i}$ 's are in $K[z]$, the $p_{i}$ 's being monic and irreducible; degree $A_{i, j}$ and degree $B_{i, j}$ are both less than degree $p_{i}$; $B_{i, 0} / p_{i}=\sum_{j=1}^{r_{i}} c_{i j} q_{i, j}^{i} / q_{i, j} i=1, \ldots, s$ where $p_{i}=\prod_{j=1}^{r_{i}} q_{i, j}$ is the factorization of $p_{i}$ into monic, irreducible (and therefore linear) factors over $\bar{K}$. 
We obtain the following conditions on the $B$ 's:

$$
\begin{aligned}
& A_{k}=(k+1) B_{k+1} \\
& \vdots \\
& A_{0}= B_{1} \\
& 0= B_{0}^{\prime} \\
& \sum_{j=1}^{k_{1}} \frac{A_{1, j}}{p_{1}^{j}}=\left[\sum_{j=1}^{k_{1}-1} \frac{B_{1, j}}{p_{1}^{j}}+\int \frac{B_{1,0}}{p_{1}}\right]^{\prime}
\end{aligned}
$$

giving $A_{1, k_{1}} \equiv-\left(k_{1}-1\right) p_{1}^{\prime} B_{1, k_{1}-1}\left(p_{1}\right)$. By $[14$, p. 88], we can uniquely solve for $R$ and $S$ when they are subjected to the conditions; $R p+S p^{\prime}=A_{1, k_{1}}, R, S$ els $K[z]$, degree $R<$ degree $p_{1}^{\prime}$, degree $S<$ degree $p_{1}$. Thus, setting

$$
-\left(k_{1}-1\right) B_{1, k_{1}-1}=S \text {, }
$$

we determine $B_{1, k_{1}-1}$. We substitute $B_{1, k_{1}-1}$ into $(+)$ and obtain a new relation:

$$
\sum_{j=1}^{k_{1}-1} \frac{A_{1 j}^{(*)}}{p_{1}^{j}}=\left[\sum_{j=1}^{k_{1}-2} \frac{B_{1, j}}{p_{1}^{j}}+\int \frac{B_{1,0}}{p_{1}}\right]^{\prime} .
$$

Continuing in this manner, we determine $B_{1, k_{1}-2}, \ldots, B_{1,1}$. Next $A_{1,1}^{(* * . .)}=B_{1,0}$ which yields

$$
\frac{A_{1,1}^{(* * \ldots)}}{p_{1}}=\sum_{j=1}^{r_{1}} c_{1, j} \frac{q_{1, j}^{\prime}}{q_{1, j}}=\sum_{j=1}^{r_{1}} \frac{c_{1, j}}{q_{1, j}}
$$

and we uniquely determine the $c_{1, j}$ 's by breaking up $A_{1,1}^{(* * . .)} / p_{1}$ into partial fractions over $\bar{K}$.

The other $B_{i, j}$ 's are determined in the same manner. We note that the conditions put on the $B$ 's are always satisfiable so $\int f$ is always elementary-as is well known. Note that no polynomial need be factored over $\bar{K}$ until the last moment. This is important in practical considerations $\left({ }^{2}\right)$.

It should be noted that we can use the above conditions to tell whether the integral is in a certain definite form. E.g., to see whether there is a $v_{0} \in \mathscr{F}$ such that $v_{0}^{\prime}=f$, we set $B_{1,0}=0, \ldots, B_{s, 0}=0$ and obtain the necessary and sufficient conditions $A_{1,1}^{(* * . .)}=0, \ldots, A_{s, 1}^{(* * . .)}=0$.

To determine for a given $v_{1} \in \mathscr{F}$ whether there is a $v_{0} \in \mathscr{F}$ and a $c \in K$ such that

(2) Professor George Collins of the University of Wisconsin has pointed out to us that we can refine the integration algorithm so as to avoid factoring into irreducibles until the logs are to be determined. Using the Euclidean algorithm we can factor any polynomial in $K(z)$ as follows: $P(z)=q_{1} q_{2}^{2} \cdots q_{k}^{k}$ where the $q_{i}$ are relatively prime and each is a product of irreducible polynomials. Everything goes through when we replace the irreducible $p_{i}$ 's used above with the $q_{i}$ 's. It is an interesting open question to determine whether we can prove the main theorem, sans the last sentence of (a), with the added stipulation that only the rational operations need to be applied to elements of $\mathscr{F}$ in performing the decision method. Proposition 1.1 indicates that we cannot actually find the $v_{i}, i>0$ without factoring over $\bar{K}$. 
$\left(v_{0}+c \log v_{1}\right)^{\prime}=f$, we write $v_{1}=a \prod_{i=1}^{s} p_{i}^{j_{i}}, a \in K, j_{i} \in Z$. Then $\log v_{1}=\sum_{i} j_{i} \int\left(p_{i}^{\prime} / p_{i}\right)$. $v_{i}$ can have no other irreducible factors besides the $p_{i}$ in the denominator of $f$. We plug in $c j_{i} p_{i}$ for each $B_{i, 0}$ and see if we can satisfy $A_{i, 1}^{(* * .)}=c j_{i} p_{i}^{\prime}, i=1, \ldots, s$. (b) We set

$$
y=\frac{A}{p_{1}^{\alpha_{1}} \cdots p_{k}^{\alpha_{k}}} \quad f=\frac{B}{p_{1}^{\beta_{1} \cdots} \cdot p_{k}^{\beta_{k}}} \quad \sum_{j=1}^{m} c_{j} g_{j}=\frac{C}{p_{1}^{\gamma} \cdots \cdot p_{k}^{\gamma_{k}}}
$$

where $p_{i}$ is a monic, irreducible element of $K[z],-\alpha_{i}=\operatorname{ord}_{p_{i}} y,-\beta_{i}=\operatorname{ord}_{p_{i}} f$, $-\gamma_{i}=\operatorname{Min}_{j}$ ord $_{p_{i}} g_{i} ; A, B, C$ els $K[z]$.

The $\beta_{i}$ and $\gamma_{i}$ are given to us. We will show that $\alpha_{i}>0$ implies that $\beta_{i}>0$ or $\gamma_{i}>0$ and find a bound, which is independent of the choice of the $c_{j}$, for each $\alpha_{i}>0$.

The $p_{i}$-adic expansion of $y$ begins: $A_{\alpha_{i}} / p_{i}^{\alpha_{i}}+\cdots$.

The $p_{i}$-adic expansion of $f$ begins: $B_{\beta_{i}} / p_{i}^{\beta_{i}}+\cdots$.

The $p_{i}$-adic expansion of $\sum_{j=1}^{m} c_{j} g_{j}$ begins: $C_{y_{i}} / p_{i}^{\gamma_{i}}+\cdots$ where the first nonzero term, in this last expansion, depends on the choice of the $c_{j}$.

Thus, by plugging these expansions into $y^{\prime}+f y=\sum c_{j} g_{j}$ we get

$$
-\alpha_{i} p_{i}^{\prime} A_{\alpha_{i}} / p_{i}^{\alpha_{i}+1}+\cdots+B_{\beta_{i}} A_{\alpha_{i}} / p_{i}^{\alpha_{i}+\beta_{i}}+\cdots=C_{\gamma_{i}} / p_{i}^{\gamma_{i}}+\cdots .
$$

Note that $p_{i} \nmid-\alpha_{i} p_{i}^{\prime} A_{\alpha_{i}}$ and $p_{i} \nmid B_{\beta_{i}} A_{\alpha_{i}}$. Looking at the highest power of $1 / p_{i}$ we must have either

$$
\alpha_{i}+1 \leqq \gamma_{i}, \quad \alpha_{i}+\beta_{i} \leqq \gamma_{i}, \quad \text { or } \quad \alpha_{i}+1=\alpha_{i}+\beta_{i}>\gamma_{i} .
$$

In the third case $-\alpha_{i} p_{i}^{\prime} A_{\alpha_{i}}+B_{\beta_{i}} A_{\alpha_{i}} \equiv 0\left(p_{i}\right),-\alpha_{i} p_{i}^{\prime}+B_{\beta_{i}}=0$.

Thus, we set, $\alpha_{i}^{*}=\operatorname{Max}\left(\gamma_{1}-1, \gamma_{i}-\beta_{i}, B_{\beta_{i}} / p_{i}^{\prime}\right.$ if this is an integer). Then $y=$ $Y / p_{1}^{\alpha_{1}^{*}} \cdots p_{k}^{\alpha_{k}^{*}}=Y / P$ where $Y$ and $P$ are in $K[z]$.

Substituting this expression for $y$ into $y^{\prime}+f y=\sum_{i=1}^{m} c_{i} g_{i}$ and clearing denominators we find $R, S, T_{i}$ in $K[z]$, which do not depend on the $c_{i}$, such that $y^{\prime}+f y$ $=\sum_{i=1}^{m} c_{i} g_{i}$ iff $\left(^{*}\right): R Y^{\prime}+S Y=\sum_{i=1}^{m} c_{i} T_{i}$. Let

$$
\begin{aligned}
Y & =y_{\alpha} z^{\alpha}+y_{\alpha-1} z^{\alpha-1}+\cdots+y_{0}, \\
R & =r_{\beta} z^{\beta}+\cdots+r_{0}, \\
S & =s_{\gamma} z^{\gamma}+\cdots+s_{0}, \\
\sum_{j=1}^{m} c_{j} T_{j} & =t_{\delta} z^{\delta}+\cdots+t_{0},
\end{aligned}
$$

where $r_{\beta}, s_{\gamma} \neq 0$, and $\delta=\operatorname{Max}_{j=1}^{m}$ (degree $T_{j}$ ), $t_{i}$ is a linear homogeneous element of $K\left[c_{1}, \ldots, c_{m}\right]$.

By substituting in $\left(^{*}\right)$ we get:

$$
\left(r_{\beta} z^{\beta}+\cdots\right)\left(\alpha y_{\alpha} z^{\alpha-1}+\cdots\right)+\left(s_{\gamma} z^{\gamma}+\cdots\right)\left(y_{\alpha} z^{\alpha}+\cdots\right)=\left(t_{\delta} z^{\delta}+\cdots\right) .
$$

When definite values in $K$ are chosen for $c_{1}, \ldots, c_{m}$ we must have

$$
\alpha+\beta-1 \leqq \delta, \quad \alpha+\gamma \leqq \delta, \quad \text { or } \quad \alpha+\beta-1=\alpha+\gamma>\delta .
$$


If the third case holds, then $\alpha y_{\alpha} r_{\beta}+y_{\alpha} s_{\gamma}=0$. Let $\alpha=r=\operatorname{Max}(\delta-\beta+1, \delta-\gamma$, $-s_{\gamma} / r_{\beta}$ if this is an integer). Take $h_{i}=z^{i} / P, i=0, \ldots, r$. By multiplying ( $\dagger$ ) out and equating powers of $z$, we get our simultaneous system $\mathscr{S}$ which satisfies the conditions of part (b) of this theorem.

Induction step. We assume that both (a) and (b) of our theorem hold for $\mathscr{D}=K\left(z, \theta_{1}, \ldots, \theta_{n-1}\right)$ and prove it for $\mathscr{F}=\mathscr{D}(\theta), \theta=\theta_{n}$. Besides (a) as stated, we assume that the simpler variants, which occur when some of the $c_{i}$ and $v_{i}$ are given, have been established-see the discussion at the end of the proof of (a) for the $K(z)$ case.

(a) Case 1. $\theta=\log \eta$. We again apply the Strong Liouville Theorem and the breaking up of $f$ and the $v_{i}$ 's into partial fractions to obtain:

$$
f=\left\{\begin{array}{c}
A_{k} \theta^{k}+\cdots+A_{0} \\
+\frac{A_{1, k_{1}}}{p_{1}^{k_{1}}}+\cdots+\frac{A_{1,1}}{p_{1}} \\
\vdots \\
+\frac{A_{s, k_{s}}}{p_{s}^{k_{s}}}+\cdots+\frac{A_{s, 1}}{p_{s}}
\end{array}\right\}=\left\{\begin{array}{c}
B_{k+1} \theta^{k+1}+\cdots+B_{0}+\sum_{j} d_{j} \log D_{j} \\
+\frac{B_{1, k_{1}-1}}{p_{1}^{k_{1}-1}}+\cdots+\frac{B_{1,1}}{p_{1}}+\int \frac{B_{1,0}}{p_{1}} \\
\vdots \\
+\frac{B_{s, k_{s}-1}}{p_{s}^{k_{s}-1}}+\cdots+\frac{B_{s, 1}}{p_{s}}+\int \frac{B_{s, 0}}{p_{s}}
\end{array}\right\}
$$

where the $A_{i}$ 's, $B_{i}$ 's and $D_{j}$ 's are in $\mathscr{D}$; the $A_{i j}$ 's, $B_{i j}$ 's and $p_{i}$ 's are in $\mathscr{D}[\theta]$, the $p_{i}$ 's being monic and irreducible; degree $A_{i j}$ and degree $B_{i j}$ are both less than the degree of $p_{i} ; B_{i, 0} / p_{i}=\sum_{j=1}^{r_{i}} c_{i j} q_{i j}^{\prime} / q_{i, j}, i=1, \ldots, s$ where $p_{i}=\prod_{j=1}^{r_{i}=1} q_{i, j}$ is the factorization of $p_{\imath}$ into monic, irreducible factors over $\bar{K} \mathscr{D} ; d_{j}$ els $\bar{K}$.

The discussion (page 169) preceding Liouville's Theorem tells us precisely what terms on the right-hand side will differentiate into each of the $A$ 's. Thus, we obtain the following relations for the $B$ 's:

$$
\begin{gathered}
0=B_{k+1}^{\prime} . \quad \text { So } B_{k+1} \in K . \\
A_{k}=(k+1) B_{k+1} \eta^{\prime} / \eta+B_{k}^{\prime} . \quad \text { Thus } \int A_{k}=B_{k}+(k+1) B_{k+1} \log \eta .
\end{gathered}
$$

By the induction assumption, we can determine whether there is a $B_{k} \in \mathscr{D}$ and $B_{k+1}$ in $K$ such that the last relation holds. $B_{k+1}$ will be uniquely determined since $\log \eta=\theta$ is a monomial over $\mathscr{D} . B_{k}$ is determined up to an additive constant. We set $B_{k}=\bar{B}_{k}+b_{k}$ where $\bar{B}_{k}$ is fixed.

$$
A_{k-1}=k B_{k} \eta^{\prime} / \eta+B_{k-1}^{\prime}
$$

Thus $\int\left(A_{k-1}-k \bar{B}_{k} \eta^{\prime} / \eta\right)=B_{k-1}+k b_{k} \log \eta$. As before we determine $b_{k} \in K$ and $B_{k-1}$ up to an additive constant, etc.

Thus

$$
A_{0}=B_{1} \eta^{\prime} / \eta+B_{0}^{\prime}+\sum d_{j}\left(\log D_{j}\right)^{\prime}
$$

$$
\int\left(A_{0}-\bar{B}_{1} \eta^{\prime} / \eta\right)=B_{0}+b_{1} \log \eta+\sum d_{j} \log D_{j}
$$

This last condition is simply that $\int\left(A_{0}-\bar{B}_{1} \eta^{\prime} / \eta\right)$ is elementary over $\mathscr{D}$. 
We determine the $B_{i, j}$ 's as in the $K(z)$ case. Then $A_{i, 1}^{(* * . .)}=B_{i, 0}$ giving $A_{i, 1}^{(* * . .)} / p_{i}$ $=\sum_{j=1}^{r_{i}} c_{i, j} q_{i, j}^{\prime} / q_{i, j}$. The $c_{i, j}$ are uniquely determined, if they exist, from the partial fraction decomposition of $A_{i, 1}^{(* * . .)} / p_{i}$.

We note that if in the course of this investigation we find that one of these conditions is not satisfied, then $f$ does not have an elementary indefinite integral and we can terminate the proceedings, right there.

Case 2. $\theta=\exp ^{\zeta}$.

$$
f=\left\{\begin{array}{l}
\left.\begin{array}{c}
A_{k} \theta^{k}+\cdots+A_{1} \theta \\
+A_{-m} \theta^{-m}+\cdots+A_{-1} \theta^{-1}+A_{0} \\
+\frac{A_{1, k_{1}}}{p_{1}^{k_{1}}}+\cdots+\frac{A_{1,1}}{p_{1}} \\
\vdots \\
+\frac{A_{s, k_{s}}}{p_{s}^{k_{s}}}+\cdots+\frac{A_{s, 1}}{p_{s}}
\end{array}\right\}=\left\{\begin{array}{l}
B_{k} \theta^{k}+\cdots+B_{1} \theta \\
+B_{-m} \theta^{-m}+\cdots+B_{-1} \theta^{-1}+B_{0}+\sum d_{j} \log D_{j} \\
+\frac{B_{1, k_{1}-1}}{p_{1}^{k_{1}-1}}+\cdots+\frac{B_{1,1}}{p_{1}}+\int \frac{B_{1,0}}{p_{1}} \\
\vdots \\
+\frac{B_{s, k_{s}-1}}{p_{s}^{k_{s}-1}}+\cdots+\frac{B_{s, 1}}{p_{s}}+\int \frac{B_{s, 0}}{p_{s}}
\end{array}\right\}
\end{array}\right\}
$$

Here, as usual, $B_{i, 0} / p_{i}=\sum_{j=1}^{r_{i}} c_{i, j} q_{i j}^{\prime} / q_{i j}$. The conditions obtained are

$$
\begin{aligned}
A_{k}= & B_{k}^{\prime}+k \zeta^{\prime} B_{k} \\
& \vdots \\
A_{1}= & B_{1}^{\prime}+\zeta^{\prime} B_{1} \\
A_{-1}= & B_{-1}^{\prime}-\zeta^{\prime} B_{-1} \\
& \vdots \\
A_{-m}= & B_{-m}^{\prime}-m \zeta^{\prime} B_{-m} .
\end{aligned}
$$

By our induction assumption for (b) of our theorem, we can write $B_{i}=\sum_{j} y_{j i} T_{j i}$, $T_{j i} \in \mathscr{D}$ where the $y_{j i}$ satisfy a linear system $\mathscr{S}$. This must determine each $B_{i}$ uniquely since two solutions would yield that the homogeneous equation $B_{i}^{\prime}+i \zeta^{\prime} B_{i}=0$ has solutions in $\mathscr{D}$. This yields a contradiction, as is well known to the reader.

We determine $B_{1, k_{1}-1}, \ldots, B_{1,1} ; B_{2, k_{2}-1}, \ldots, B_{2,1} ; . . \cdot ; B_{s, k_{s}-1}, \ldots, B_{s, 1}$ in the same manner as previously. Then we obtain:

$$
\sum_{i=1}^{s} \frac{A_{i, 1}^{(* * . .)}}{p_{i}}+A_{0}=\sum_{i=1}^{s} \frac{B_{i, 0}}{p_{i}}+B_{0}^{\prime}+\left(\sum d_{j} \log D_{j}\right)^{\prime}
$$

From this, $A_{i, 1}^{(* * .)} \equiv B_{i, 0}\left(p_{i}\right)$. Let degree of $q_{i, j}=n_{i}$. Then

$$
\frac{A_{i, 1}^{(* * \ldots)}+n_{i} \zeta^{\prime}\left(\sum_{j=1}^{r_{i}} c_{i j}\right) p_{i}}{p_{i}}=\frac{B_{i, 0}}{p_{i}}=\sum_{j=1}^{r_{i}} c_{i j} \frac{q_{i, j}^{\prime}}{q_{i, j}} .
$$

We determine whether such $c_{i j}$ exist, and find them if they do, from consideration of the partial fraction decomposition of the functions in this equation.

Then $A_{0}-\zeta^{\prime} \sum_{i, j} n_{i} c_{i, j}=\left(B_{0}+\sum d_{j} \log D_{j}\right)^{\prime}$ and our problem is reduced to telling whether $\int\left(A_{0}-\zeta^{\prime} \sum_{i, j} n_{i} c_{i, j}\right)$ is elementary over $\mathscr{D}$.

(b) Here the general method is the same as that for the $K(z)$ case. The problem of determining bounds is more difficult. 
Case 1. $\theta=\log \eta$. For $y=A / p_{1}^{\alpha}{ }^{1} \cdots p_{k}^{\alpha}$ we determine the bounds $\alpha_{i}^{*}, i-1, \ldots, k$ in the same way as in the $K(z)$ case. So we have $y=Y / p_{1}^{\alpha_{1}^{*}} \cdots p_{k}^{\alpha_{k}^{*}}=Y / P, Y, P$ els of $\mathscr{D}[\theta]$. As before we reduce to the equation:

$$
R Y^{\prime}+S Y=\sum_{j=1}^{m} c_{j} T_{j}
$$

We set

$$
\begin{aligned}
& Y=y_{\alpha} \theta^{\alpha}+y_{\alpha-1} \theta^{\alpha-1}+\cdots+y_{0}, \\
& R=r_{\beta} \theta^{\beta}+\cdots+r_{0}, \\
& S=s_{\gamma} \theta^{\gamma}+\cdots+s_{0}, \\
& \sum_{j=1}^{m} c_{j} T_{j}=t_{\delta} \theta^{\delta}+\cdots+t_{0}, \quad y_{\alpha}, r_{\beta}, s_{\gamma} \neq 0, \delta=\operatorname{Max}_{i=1}^{m}\left(\operatorname{degree} T_{i}\right) .
\end{aligned}
$$

Each $y_{j}, r_{j}, s_{j}$ is in $\mathscr{D}, t_{j}$ is a linear homogeneous element of $\mathscr{D}\left[c_{1}, \ldots, c_{m}\right]$. Substitution in (1) yields

$$
\begin{aligned}
& \left(r_{\beta} \theta^{\beta}+r_{\beta-1} \theta^{\beta-1}+\cdots\right)\left(y_{\alpha}^{\prime} \theta^{\alpha}+\left(y_{\alpha-1}^{\prime}+\alpha\left(\eta^{\prime} / \eta\right) y_{\alpha}\right) \theta^{\alpha-1}+\cdots\right) \\
& +\left(s_{\gamma} \theta^{\gamma}+s_{\gamma-1} \theta^{\gamma-1}+\cdots\right)\left(y_{\alpha} \theta^{\alpha}+y_{\alpha-1} \theta^{\alpha-1}+\cdots\right)=t_{\delta} \theta^{\delta}+\cdots+t_{0}
\end{aligned}
$$

For definite values of $c_{1}, \ldots, c_{m}$ chosen in $K$, we get

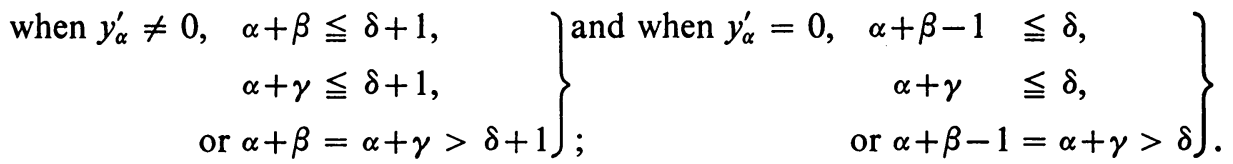

When $y_{\alpha}^{\prime} \neq 0$ the third condition occurs when $r_{\beta} y_{\alpha}^{\prime}+s_{\gamma} y_{\alpha}=0$ and $r_{\beta} y_{\alpha-1}^{\prime}+s_{\gamma} y_{\alpha-1}$ $+r_{\beta-1} y_{\alpha}^{\prime}+\left(\alpha\left(\eta^{\prime} / \eta\right) r_{\beta}+s_{\gamma-1}\right) y_{\alpha}=0$.

Letting $y_{\alpha-1}=v y_{\alpha}, v \in \mathscr{D}$ we have that

$$
\begin{gathered}
r_{\beta} y_{\alpha} v^{\prime}+\left(r_{\beta} y_{\alpha}^{\prime}+s_{\gamma} y_{\alpha}\right) v+r_{\beta-1} y_{\alpha}^{\prime}+\left(s_{\gamma-1}+\alpha\left(\eta^{\prime} / \eta\right) r_{\beta}\right) y_{\alpha}=0, \\
v^{\prime}-r_{\beta-1} s_{\gamma} / r_{\beta}^{2}+s_{\gamma-1} / r_{\beta}+\alpha\left(\eta^{\prime} / \eta\right)=0, \\
\int \frac{r_{\beta-1} s_{\gamma}-r_{\beta} s_{r-1}}{r_{\beta}^{2}}=v+\alpha \log \eta .
\end{gathered}
$$

If $y_{\alpha}^{\prime}=0$, the third case occurs when $r_{\beta}\left(y_{\alpha-1}^{\prime}+\alpha\left(\eta^{\prime} / \eta\right) y_{\alpha}\right)+s_{y} y_{\alpha}=0$.

$$
\int \frac{s_{y}}{r_{\beta}}=\frac{-y_{\alpha-1}}{y_{\alpha}}-\alpha \log \eta
$$

In both instances the induction assumption for part (a) of our Main Theorem tells us we can determine the constant $\alpha$ so that the integral is of this form. We let $\mu=\operatorname{Max}(\delta+1-\beta ; \delta+1-\gamma$; the $\alpha$ 's determined in the third case, if they are integers). 
We now rewrite (2) in the form:

$$
\begin{aligned}
&\left(r_{\lambda} \theta^{\lambda}+r_{\lambda-1} \theta^{\lambda-1}+\cdots\right)\left(y_{\mu}^{\prime} \theta^{\mu}+\left(y_{\mu-1}^{\prime}+\mu\left(\eta^{\prime} / \eta\right) y_{\mu}\right) \theta^{\mu-1}+\cdots\right) \\
&+\left(s_{\lambda} \theta^{\lambda}+s_{\lambda-1} \theta^{\lambda-1}+\cdots\right)\left(y_{\mu} \theta^{\mu}+y_{\mu-1} \theta^{\mu-1}+\cdots\right) \\
&=t_{\lambda+\mu} \theta^{\lambda+\mu}+\cdots+t_{0}
\end{aligned}
$$

so that at least one of $r_{\lambda}, s_{\lambda}$, and $t_{\lambda+\mu}$ is not equal to zero.

We complete the proof of Case 1 by showing, by induction on $\mu$, that there is a simultaneous system of linear algebraic equations, $\mathscr{S}$, in $m+p+q$ variables, with coefficients in $K$, and $h_{1}, \ldots, h_{p}, j_{1}, \ldots, j_{q}$ in $\mathscr{D}[\theta]$, all of which are of degree $\leqq \mu$, such that $\left(y_{\mu} \theta^{\mu}+\cdots\right)$ satisfies (3) iff $\left(y_{\mu} \theta^{\mu}+\cdots\right)=\sum_{i=1}^{p} d_{i} h_{i}+\sum_{i=1}^{q} e_{i} j_{i}$ where $d_{i}, e_{i}$ are in $K$ and $c_{1}, \ldots, c_{m}, d_{1}, \ldots, d_{p}, e_{1}, \ldots, e_{q}$ satisfy $\mathscr{S}$. The functions in the statement of (b) of the Main Theorem will be $h_{1} / P, \ldots, j_{q} / P$.

In this induction we assume $r_{\lambda}$ or $s_{\lambda} \neq 0$. If $r_{\lambda}=s_{\lambda}=\cdots=r_{\lambda-l+1}=s_{\lambda-l+1}=0$ while $r_{\lambda-l}$ or $s_{\lambda-l} \neq 0$, then the system

$$
\begin{aligned}
& t_{\lambda}=0 \\
& \vdots \\
& t_{\lambda-l-1}=0
\end{aligned}
$$

is equivalent, by the lemma, to a linear system in the $c_{i}$ with coefficients in $K$. Then we proceed as below with $r_{\lambda-l}, s_{\lambda-l}$ taking the place of $r_{\lambda}$ and $s_{\lambda}$.

$\mu=0$. Here (3) takes the form

$$
\left(r_{\lambda} \theta^{\lambda}+\cdots\right) y_{0}^{\prime}+\left(s_{\lambda} \theta^{\lambda}+\cdots\right) y_{0}=\left(t_{\lambda} \theta^{\lambda}+\cdots\right) .
$$

Looking at the coefficient of $\theta^{\lambda}$, we get

$$
r_{\lambda} y_{0}^{\prime}+s_{\lambda} y_{0}=t_{\lambda} \text {. }
$$

By the induction assumption for $n-1$ for (b) we get that (5) is equivalent to $y_{0}=\sum_{i=1}^{r} d_{i} h_{i}, h_{i}$ els $\mathscr{D}$, and a linear system $\mathscr{S}^{\prime}$ with coefficients in $K$. Plugging this expression for $y_{0}$ into the remaining $\lambda$ equations for $y_{0}$ which can be obtained from (4), yields a simultaneous set of linear algebraic equations in $d_{1}, \ldots, d_{p}, c_{1}, \ldots, c_{m}$ with coefficients in $\mathscr{D}$, which by the lemma to this theorem is equivalent to a linear system $\mathscr{S}^{\prime \prime}$ with coefficients in $K$, in the same variables. Take $\mathscr{S}=\mathscr{S}^{\prime} \cup \mathscr{S}^{\prime \prime}$ and $j_{i}=0, i=1, \ldots, q$ and we have our result for $\mu=0$.

Assume the result is true for $\mu-1$. Then the leading coefficient $y_{\mu}$ satisfies

$$
r_{\lambda} y_{\mu}^{\prime}+s_{\lambda} y_{\mu}=t_{\lambda+\mu} \text {. }
$$

By the induction assumption for $n-1$ for part (b) we have that (6) is equivalent to $y_{\mu}=\sum_{i=1}^{p} d_{i} h_{i}, h_{i}$ els $\mathscr{D}$ and a linear algebraic system $\mathscr{S}^{\prime}$ in $c_{1}, \ldots, c_{m}, d_{1}, \ldots, d_{p}$. Substituting the expression for $y_{\mu}$ into (3) we get

$$
\begin{aligned}
\left(r_{\lambda} \theta^{\lambda}+\cdots\right) & {\left[y_{\mu-1}^{\prime} \theta^{\mu-1}+\left(y_{\mu-2}^{\prime}+(\mu-1)\left(\eta^{\prime} / \eta\right) y_{\mu-1}\right) \theta^{\mu-2}+\cdots\right] } \\
& +\left(s_{\lambda} \theta^{\lambda}+s_{\lambda-1} \theta^{\lambda-1}+\cdots\right)\left[y_{\mu-1} \theta^{\mu-1}+y_{\mu-2} \theta^{\mu-2}+\cdots\right] \\
= & \left(u_{\lambda+\mu-1} \theta^{\lambda+\mu-1}+\cdots\right)
\end{aligned}
$$

where the $u_{i}$ 's are linear homogeneous elements of $K\left[c_{1}, \ldots, c_{m}, d_{1}, \ldots, d_{p}\right]$. 
By our induction assumption for $\mu-1$, we have that there are $j_{1}, \ldots, j_{q}$ in $\mathscr{D}[\theta]$, with degree $j_{i} \leqq \mu-1$ such that (7) is equivalent to $\left(y_{\mu-1} \theta^{\mu-1}+\cdots\right)=\sum_{i=1}^{q} e_{i} j_{i}$ and a linear algebraic system $\mathscr{S}^{\prime \prime}$ in $c_{1}, \ldots, c_{m}, d_{1}, \ldots, d_{p}, e_{1}, \ldots, e_{q}$. We take $\mathscr{S}=\mathscr{S}^{\prime} \cup \mathscr{S}^{\prime \prime}$ and by writing $\left(y_{\mu} \theta^{\mu}+\cdots\right)=\sum_{i=1}^{p} d_{i} h_{i} \theta^{\mu}+\sum_{i=1}^{q} e_{i} j_{i}$ we have found conditions equivalent to (3). This completes our induction on $\mu$.

Case 2. $\theta=\exp \zeta$. Here, in distinction to the $K(z)$ case and Case 1 , degree $p_{i}^{\prime}$ $=$ degree $p_{i}$ so things are slightly different. We set

$$
y=\frac{A}{p_{1}^{\alpha} \cdots p_{k}^{\alpha} \alpha_{k}^{\alpha}}, \quad f=\frac{B}{p_{1}^{\beta_{1}} \cdots p_{k}^{\beta}}, \quad \sum_{j=1}^{m} c_{j} g_{j}=\frac{C}{p_{1}^{\gamma_{1}} \cdots p_{k^{\gamma}}^{\gamma_{k}}},
$$

and show that $\alpha_{i}>0$ implies that either $\beta_{i}>0, \gamma_{i}>0$, or $p_{i}=\theta$ and we find a bound for each $\alpha_{i}$.

Looking at the $p_{i}$-adic expansion of the terms involved in $y^{\prime}+f y=\sum_{j=1}^{m} c_{j} g_{j}$, we get if $p_{i} \nmid p_{i}^{\prime}$ :

$$
-\alpha_{i} p_{i}^{\prime} A_{\alpha_{i}} / p_{i}^{\alpha_{i}+1}+\cdots+B_{\beta_{i}} A_{\alpha_{i}} / p_{i}^{\alpha_{i}+\beta_{i}}+\cdots=C_{\gamma_{i}} / p_{i}^{\gamma_{i}} .
$$

Since $p_{i} \nmid-\alpha_{i} p_{i}^{\prime} A_{\alpha_{i}}$, we have that either

$$
\alpha_{i}+1 \leqq \gamma_{i}, \quad \alpha_{i}+\beta_{i} \leqq \gamma_{i}, \quad \text { or } \quad \alpha_{i}+1=\alpha_{i}+\beta_{i}>\gamma_{i} .
$$

In the latter case

$$
\begin{aligned}
-\alpha_{i} p_{i}^{\prime} A_{\alpha_{i}}+B_{\beta_{i}} A_{\alpha_{i}} & \equiv 0\left(p_{i}\right), \\
-\alpha_{i} p_{i}^{\prime}+B_{\beta_{i}} & \equiv 0\left(p_{i}\right), \\
-\alpha_{i} p_{i}^{\prime}+B_{\beta_{i}} & =-\alpha_{i} n_{i} \zeta^{\prime} p_{i} \quad \text { where } n_{i}=\text { degree } p_{i} .
\end{aligned}
$$

So we set here

$$
\alpha_{i}^{*}=\operatorname{Max}\left(\gamma_{i}-1, \gamma_{i}-\beta_{i}, B_{\beta_{i}} /\left(p_{i}^{\prime}-n_{i} \zeta^{\prime} p_{i}\right)\right. \text { if this is an integer). }
$$

$p_{i} \mid p_{i}^{\prime}$ iff $p_{i}=\theta$ (see page 169). Thus, here, the $p_{i}$-adic expansion runs

$$
\frac{A_{\alpha_{i}}^{\prime}-\alpha_{i} \zeta^{\prime} A_{\alpha_{i}}}{p_{i}^{\alpha_{i}}}+\cdots+\frac{B_{\beta_{i}} A_{\alpha_{i}}}{p_{i}^{\alpha_{i}+\beta_{i}}}+\cdots=\frac{C_{\gamma_{i}}}{p_{i}^{\gamma_{i}}}
$$

Then

$$
\alpha_{i} \leqq \gamma_{i}, \quad \alpha_{i}+\beta_{i} \leqq \gamma_{i}, \quad \text { or } \quad \alpha_{i}=\alpha_{i}+\beta_{i}>\gamma_{i} .
$$

In the third case $\left(A_{\alpha_{i}}^{\prime} / A_{\alpha_{i}}\right)-\alpha_{i} \zeta^{\prime}+B_{0}=0$ and by our induction hypothesis (for (a)) we determine if there is an $\alpha \in K$ and an $A_{\alpha_{i}}$ in $\mathscr{D}$ such that $\int B_{0}=\alpha_{i} \zeta-\log A_{\alpha_{i}}$. We take here

$$
\alpha_{i}^{*}=\operatorname{Max}\left(\gamma_{i}, \gamma_{i}-\beta_{i} \text {, the } \alpha_{i}\right. \text { determined in the third case if it is an integer). }
$$

Setting $y=Y / p_{1}^{\alpha^{*}} \cdots p_{k}^{\alpha_{k}^{*}}=Y / P$ as before, we obtain the equation $R Y^{\prime}+S Y$ $=\sum_{j=1}^{m} c_{j} T_{j}$. Equation (2) of Case 1 is replaced by

$$
\left(r_{\beta} \theta^{\beta}+\cdots\right)\left[\left(y_{\alpha}^{\prime}+\alpha \zeta^{\prime} y_{\alpha}\right) \theta^{\alpha}+\cdots\right]+\left(s_{\gamma} \theta^{\gamma}+\cdots\right)\left(y_{\alpha} \theta^{\alpha}+\cdots\right)=t_{\delta} \theta^{\delta}+\cdots+t_{0} .
$$


For definite values of $c_{1}, \ldots, c_{m}$ chosen in $K$, we get,

$$
\alpha+\beta \leqq \delta, \quad \alpha+\gamma \leqq \delta, \quad \text { or } \quad \alpha+\beta=\alpha+\gamma>\delta .
$$

In the third case $r_{\beta} y_{\alpha}^{\prime}+\left(\alpha \zeta^{\prime} r_{\beta}+s_{\gamma}\right) y_{\alpha}=0$. Then $\int s_{\gamma} / r_{\beta}=-\alpha \zeta-\log y_{\alpha}$. By our induction hypothesis (for (a)) we determine if there is an $\alpha \in K$ and a $y_{\alpha}$ in $\mathscr{D}$ such that the above holds.

Thus, let $\mu=\operatorname{Max}(\delta-\beta ; \delta-\gamma$; the $\alpha$ determined in the third case, if it is an integer).

With this bound for the degree of $Y$ determined, we proceed in the same manner as in Case 1 to find the desired functions and linear system.

This completes the proof of the Main Theorem.

From this result it is easy to see how to complete the proof of the two theorems given at the beginning of $\S 3$. For (1) we must show how to decide whether a given efd in $\mathscr{E}, e=\left\langle 1_{e}, \alpha_{1}, \ldots, \delta_{1}, \ldots, \delta_{n}\right\rangle$, has an $e$ model $(\mathscr{D}, V)$ in which $V\left(\delta_{i}\right)$ is a monomial over $Q\left(V\left(\alpha_{1}\right), \ldots, V\left(\delta_{i-1}\right)\right)$. By Proposition 1.2, it suffices to be able to decide for a given $f \in \mathscr{D}$, is $\int\left(f^{\prime} \mid f\right)$ in $\mathscr{D}$ and is $\int f^{\prime}=(1 / m) \log h$ for some $m \in Z$ and $h \in \mathscr{D}$ These are simple variants of (a) of the Main Theorem.

E.g., for the problem of telling if for a given $f \in K\left(z, \theta_{1}, \ldots, \theta_{n}\right)$, where $\theta_{n}=\exp \zeta$, is $\int f^{\prime}=(1 / m) \log h, h \in K\left(z, \theta_{1}, \ldots, \theta_{n}\right), m \in Z$, we write $h=\theta_{n}^{k_{0}} p_{1}^{k_{1}} \cdots p_{r}^{k_{r}} D, p_{i}$ a monic and irreducible member of $K\left(z, \theta_{1}, \ldots, \theta_{n-1}\right)\left[\theta_{n}\right]$, degree $p_{i}=n_{i}$,

$$
D \in K\left(z, \theta_{1}, \ldots, \theta_{n-1}\right) \text {. }
$$

Then $f^{\prime}=A_{0}+A_{1} / p_{1}+\cdots+A_{r} / p_{r}=\left[q_{0} \zeta+(1 / m) \log D+q_{1} \log p_{1}+\cdots+q_{r} \log p_{r}\right]^{\prime}$ where $q_{i}=k_{i} / m$.

For $i>0, q_{i} p_{i}^{\prime} \equiv A_{i}\left(p_{i}\right)$ determines each $q_{i}$.

$$
A_{0}=q_{0} \zeta^{\prime}+\frac{1}{m} \frac{D^{\prime}}{D}+\sum_{i=1}^{r} q_{i} n_{i} \zeta^{\prime}
$$

Thus, the problem is reduced to " is $\int\left(A_{0}-\sum_{i=1}^{r} q_{i} n_{i} \zeta^{\prime}\right)=q_{0} \zeta+(1 / n) \log D$ where $q_{0} \in Q$ ?", etc.

The proof of the second theorem given at the beginning of $\$ 3$ is clear from (a) of the Main Theorem.

We now turn to some simple examples of the algorithm.

Investigate. $\int \exp z^{2}$. We show first that $\left\langle 1_{e}, z_{e}, \exp _{e} z_{e} \cdot{ }_{e} z_{e}\right\rangle$ is regular. $\int 2 z$ $=(1 / n) \log D$, for $D \in Q(z)$, is clearly impossible. Thus $\exp z^{2}$ is a monomial over $Q(z)$.

$$
\begin{aligned}
& \int \exp z^{2}=B_{1} \exp z^{2}+B_{0}, \quad B_{1}, B_{0} \text { els } Q(z) . \\
& B_{0}^{\prime}=0 \text { so } B_{0}=\text { constant. } \\
& B_{1}^{\prime}+2 z B_{1}=1, \quad B_{1} \text { must be in } K[z] \text { and thus } B_{1}=y_{\alpha} z^{\alpha}+\cdots+y_{0} . \\
& \alpha^{*}=\operatorname{Max}(1,-1, \text { third case impossible). } \\
& B_{1}=y_{1} z+y_{0} .
\end{aligned}
$$


$y_{1}+2 z\left(y_{1} z+y_{0}\right)=1$.

$\left.\begin{array}{l}2 y_{1}=0 \\ 2 y_{0}=0\end{array}\right\}$ This system can not be satisfied so the integral is not

$\left.\begin{array}{rl}2 y_{0} & =0 \\ y_{1} & =1\end{array}\right\}$ elementary.

Investigate.

$$
\int\left[2 z \exp z^{2} \log z+\frac{\exp z^{2}}{z}+\frac{\log z-2}{\left[(\log z)^{2}+z\right]^{2}}+\frac{(2 / z) \log z+1 / z+1}{(\log z)^{2}+z}\right] .
$$

First we must show that $\log z$ is a monomial over $Q\left(z, \exp z^{2}\right)$. If not, then $\int(1 / z)$ must be in $Q\left(z, \exp z^{2}\right)$. Then $\int(1 / z)=A_{0}, A_{0} \in Q(z)$. But this is clearly impossible, as we see from looking at the partial fraction decomposition of $A_{0}$.

Our integral must equal

$$
B_{2}(\log z)^{2}+B_{1} \log z+B_{0}+\Sigma d_{i} \log D_{i}+\frac{B_{1,1}}{(\log z)^{2}+z}+c_{1} \log \left[(\log z)^{2}+z\right]
$$

$B_{2}^{\prime}=0$ so $B_{2}$ is a constant. $(2 / z) B_{2}+B_{1}^{\prime}=2 z \exp z^{2}$. Thus,

$$
B_{1}=\left(\int 2 z \exp z^{2}\right)-2 B_{2} \log z=\exp z^{2}+b_{1}-2 B_{2} \log z
$$

$b_{1}$ a constant. $B_{2}=0$ since $B_{1}$ must be in $Q\left(z\right.$, exp $\left.z^{2}\right)$. Thus, $B_{1}=\exp z^{2}+b_{1}$.

$$
\begin{aligned}
\frac{\exp z^{2}}{z}+\frac{b_{1}}{z}+B_{0}^{\prime}+\left(\Sigma d_{i} \log D_{i}\right)^{\prime} & =\frac{\exp z^{2}}{z} . \\
B_{0}+\Sigma d_{i} \log D_{i}+b_{1} \log z & =\int_{0} \text { constant }
\end{aligned}
$$

so $b_{1}$ can be taken to be 0 .

$$
-B_{1,1}\left(\frac{2}{z} \log z+1\right) \equiv \log z-2 \quad\left((\log z)^{2}+z\right)
$$

We must solve $A[(2 / z) \log z+1]+B\left[(\log z)^{2}+z\right]=\log z-2$, degree $A<2$, degree $B=0$.

By the Euclidean algorithm, $A=\log z, B=-2 / z$. Thus $B_{1,1}=-\log z$. Substituting this into

$$
\frac{\log z-2}{\left[(\log z)^{2}+z\right]^{2}}+\frac{(2 / z) \log z+1 / z+1}{(\log z)^{2}+z}=\left[\frac{B_{1,1}}{(\log z)^{2}+z}+c_{1} \log \left[(\log z)^{2}+z\right]\right]^{\prime},
$$

we get $c_{1}((2 / z) \log z+1)=(2 / z) \log z+1$. Therefore, $c_{1}=1$. So our integral is

$$
\exp z^{2} \log z-\frac{\log z}{(\log z)^{2}+z}+\log \left[(\log z)^{2}+z\right]
$$




\section{REFERENCES}

1. M. Davis, Computability and unsolvability, McGraw-Hill, New York, 1958.

2. M. Davis, H. Putnam and J. Robinson, The decision problem for exponential diophantine equations, Ann. of Math. (2) 74 (1961), 425-436.

3. I. Kaplansky, An introduction to differential algebra, Hermann, Paris, 1957.

4. E. R. Kolchin, Galois theory of differential fields, Amer. J. Math. 75 (1953), 753-824.

5. W. Krull, Elementare und klassiche Algebra. Vol. II, de Gruyter, Berlin, 1959.

6. J. Liouville, Sur la detérmination des integrales dont la valeur est algébrique, J. École Polytech. 14 (1833), 124-193.

7. —_ Mémoire sir les transcendantes elliptiques de premiére et de seconde espéce, considerées comme fonctiones de leur amplitude, J. École Polytech. 14 (1833), 57-83.

8. D. D. Mordoukhay-Boltovskoy, On the integration of transcendental functions, Warsaw Univ. Izv. nos. 6-9 (1913). (Russian)

9. A. Ostrowski, Sur l'intégrabilité élémentare de quelques classes d'expressions, Comment. Math. Helv. 28 (1946), 283-308.

10. J. F. Ritt, Integration in finite terms, Liouville's theory of elementary methods, Columbia Univ. Press, New York, 1948.

11. M. Rosenlicht, Liouville's theorem on functions with elementary integrals, Pacific J. Math. 24 (1968), 153-161.

12. A. Seidenberg, Abstract differential algebra and the analytic case, Proc. Amer. Math. Soc. 9 (1958), 159-164.

13. D. M. Sintzov, Rational integrals of linear equations, Učen. Zap. Kazan Univ. 65 (1898). (Russian)

14. B. L. van der Waerden, Modern algebra. Vol. I, Ungar, New York, 1949.

System DeVelopment Corporation,

Santa Monica, California 